\title{
COMPARISON OF COLOUR CURVES OF MIRA STARS OF SPECTRUM M AND S
}

\author{
C. PAYNE-GAPOSCHKIN
}

Center for Astrophysics, Cambridge, U.S.A.

\begin{abstract}
Colour variations for Mira stars of spectrum Me and Se are deduced from the two micron survey of Neugebauer and Leighton and concurrent visual observations of the same stars.

The colours change cyclically during the periodic change of brightness. Colour is a function of maximal spectrum and also of period. Mira stars of class Se are bluer than those of Me of similar period.
\end{abstract}

A study of the light and colour curves of Mira stars, based on the infra-red catalogue of Neugebauer and Leighton and concurrent observations compiled by the American Association of Variable Star Observers, is being carried out by the writer and Charles A. Whitney. Neugebauer and Leighton tabulate magnitudes that they designate $I$ and $K$, corresponding to effective wavelengths about $0.8 \mu$ and $2.2 \mu$ respectively. Their $K$ magnitudes correspond to the $K$ magnitudes of the standard Johnson system; their $I$ magnitudes, which correspond to a shorter effective wavelength than Johnson's, (and which we accordingly designate $I^{1}$ ), are related to the Johnson system by the relation:

$$
(I-K)=0.745\left(I^{\prime}-K\right)-0.13
$$

The $I$ and $K$ magnitudes, and the visual magnitudes, which we designate $V$, although they are not exactly equivalent to $V$ magnitudes, permit us to determine three colour indices, $\left(I^{\prime}-K\right),\left(V-I^{\prime}\right)$, and $(V-K)$ for each dated observation.

Colour indices and phases (referred to visual maximum) are available for 125 Mira stars of spectrum $M$, and for 17 stars of spectrum $S$, and these form the basis of the present comparison. All the colours vary with phase, being bluest at visual maximum, reddest near visual minimum. The $\mathbf{M}$ stars are treated in three period groups (mean periods 239 , 350 and 456 days); the mean period for $S$ stars is 380 days.

Figure 1 shows colour-colour curves for the Mira stars and compares them with the colour-colour relation for non-variable stars of luminosity class III. Data for the three period groups are plotted on shifted scales, as described in the legend. On the fall from visual maximum, or slightly later, the points conform to the relation shown by the non-variable stars of the same spectral class. The colours describe counter-clockwise cycles, compatible with $I^{\prime}$ being relatively brighter on the rising curve than on the fall. When period is taken into account, it is evident that the loop for the $\mathrm{S}$ stars makes them somewhat bluer than the corresponding $M$ stars.

A synthesis of the $K$ curves for multiply observed Me and Se stars shows that the $K$ magnitude comes to maximum about 0 P after visual maximum, an effect also observed 


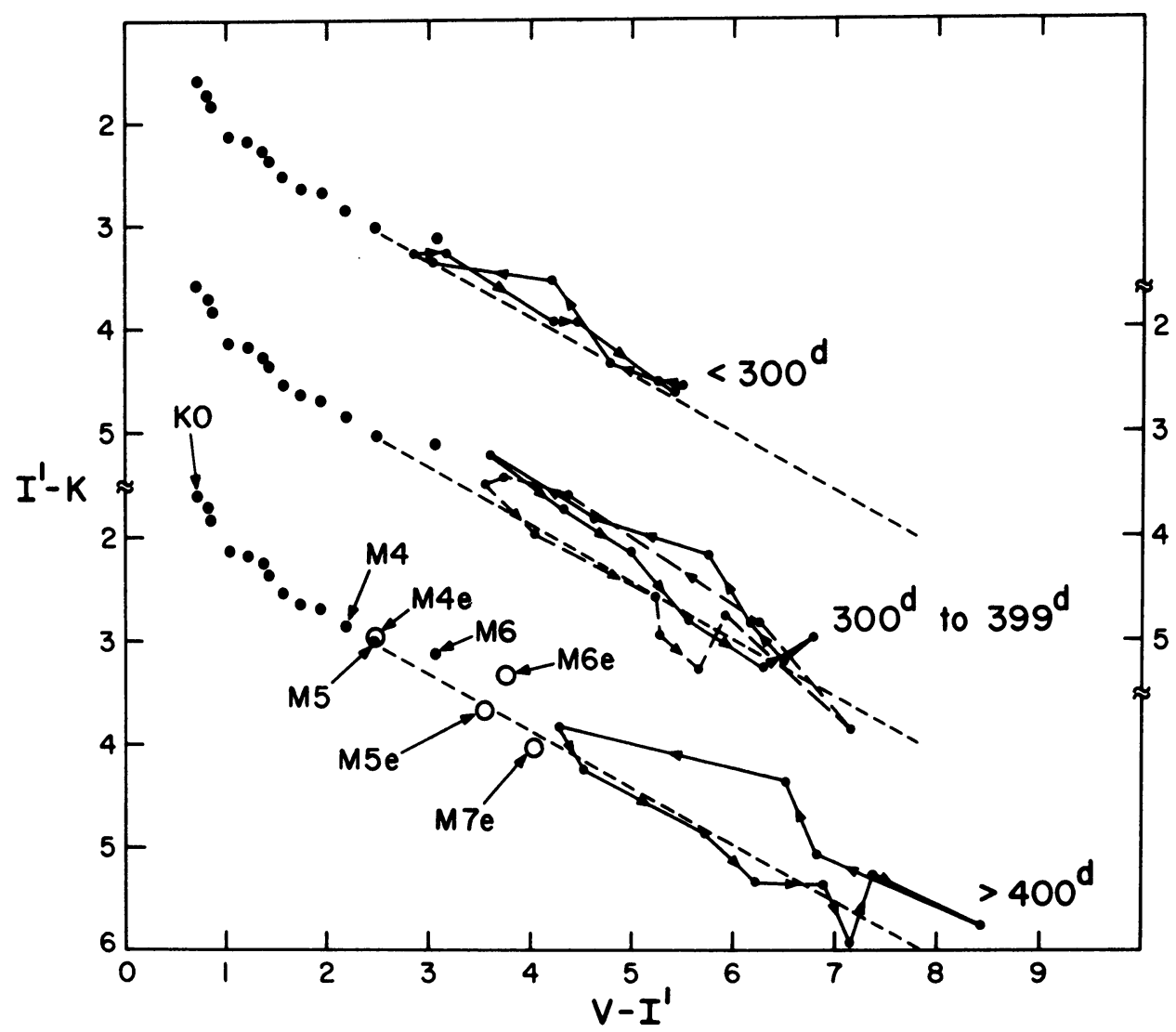

Fig 1. Colour-colour curves for non-variable stars of late type, luminosity class III, and Mira stars of spectrum $M$ and $S$. Ordinate, in magnitudes, $\left(I^{\prime}-K\right)$; abscissa, in magnitudes, $(V-I)$. Heavy dots (plotted three times on shifted scales of ordinates) are non-variable stars from K0 to M6. Smaller dots, loops described during the cycle of variation for stars of average period 239,350 , and 456 days, class Me. Broken lines, Se stars, mean period 380 days. Dotted lines extend the relation for non-variable stars through points corresponding to the fall from maximum. On the lowest curve, points corresponding to K0III, M4III, M5III and M6III are identified. Circles show the relation between maximal colours for Mira stars of classes M4e, M5e, M6e and M7e.

in the bolometric light curves determined by Pettit and Nicholson. The range of $K$ is somewhat greater for Se than for Me stars.

It is evident that the $I^{\prime}$ magnitude (and perhaps also the $V$ magnitude) is relatively brighter on the rise from minimum to bolometric (and $K$ ) maximum. Whether this excess is related to the development of the bright-line spectrum can only be decided by spectrophotometry.

Interpretation of the colours in terms of temperature requires an independent calibration. Attempts to deduce effective temperatures by means of angular diameters have led to inconsistent results when our data are compared for normal giants, supergiants, and 
Mira Ceti. Attempts to derive colour temperatures are vitiated by unknown contributions of band absorption. The fact that $\mathrm{S}$ stars are bluer in all three colour systems than $\mathrm{M}$ stars of the same period must be studied in this context.

\section{DISCUSSION}

Mendoza: How do infrared excesses, shown by some Me and Se stars, affect your results?

Payne-Gaposchkin: I believe that the fact that the Mira stars of all periods are bluer on the rise than on the fall of the light curve, is related to the greater strength of the bright line (and probably the continuum) of hydrogen as the brightness increases. A quantitative answer would require spectrophotometry. 